\title{
AVALIAÇÃO DA EFICÁCIA E SEGURANÇA DO DICLORIDRATO DE BETAISTINA EM CÃES COM DISTÚRBIOS VESTIBULARES
}

\author{
BRUM, Alexandre Martini ${ }^{1}$ \\ PASCON, João Paulo da Exaltação ${ }^{2}$ \\ CHAMPION, Tatiana ${ }^{3}$ \\ TINUCCI-COSTA, Mirela ${ }^{4}$
}

Recebido em: 2009-07-29

Aprovado em: 2009-10-14

ISSUE DOI: $10.3738 / 1982.2278 .277$

RESUMO: Doença vestibular é comum em cães e gatos, podendo ser resultado de doença central ou periférica. A patofisiologia ainda é pouco conhecida, porém pode estar relacionada à dinâmica anormal da endolinfa ou neurites da porção vestibular do VIII nervo craniano. A recuperação neurológica é longa e, em casos crônicos, os déficits neurológicos podem ser irreversíveis. O dicloridrato de betaistina é uma medicação utilizada em humanos com distúrbio vestibular periférico e foi empregada em quatros casos clínicos de cães com síndrome vestibular. Os resultados mostraram melhora clínica com sete a 10 dias de tratamento e recuperação quase completa entre 20 e 30 dias. Um ano após os referidos tratamentos os animais não apresentaram recidiva do quadro. A utilização da betaistina em cães com síndrome vestibular periférica apresentou rápida melhora clínica e ausência de efeitos adversos.

Palavras-chave: Cão. Síndrome vestibular. Betaistina

\section{EVALUATION OF EFFICACY AND SAFETY OF BETAHISTINE DIHYDROCHLORIDE IN DOGS WITH VESTIBULAR DISTURBANCES.}

SUMMARY: Vestibular disease is in dogs and cats, and it may be the result of central or peripheral disease. The pathophysiology is unknown, however it can be related to an abnormal dynamic of endolymphatic fluid or neuritis of the vestibular portion of the VIII cranial nerve. The recovery of neurological sings is slow and, in chronic cases, the neurological deficits can be irreversible. The betahistine dihydrochloride is a drug used in humans with peripheral vestibular disorders and was used in four dogs with vestibular syndrome. The results showed clinical improvement in 7 to 10 days of treatment and completed recovery in 20 to 30 days. One year after the treatment, the dogs didn't have recurrence of the syndrome. The use of betahistine dihydrochloride in dogs with peripheral vestibular syndrome shows rapid clinical recover, without laboratorial abnormalities or recurrence of the clinical signs.

Keywords: Dog. Vestibular syndrome. Betahistine

\footnotetext{
${ }^{1}$ Doutorando na UNESP/Jaboticabal, na área de nefrologia de pequenos animais e professor da UNIFRAN, nas disciplinas de Çlínica Médica e Terapêutica de Pequenos Animais e LAboratório Clínico Veterinário. E-mail: alexmbrum@bol.com.br

${ }^{2}$ Doutorando do Programa de Medicina veterinária da FCAV-UNERSP/Jaboticabal. E-mail: jpep23@yahoo.com.br

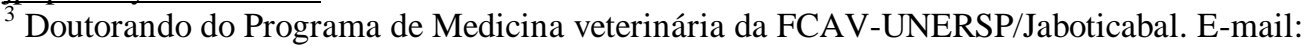
tati.cogu@ig.com.br

${ }^{4}$ Docente do Deparatmento de Clínica e Cirurgia Veterinária. E-mail: mirela@ fcav.unesp.br
} 


\section{INTRODUÇÃO}

Alterações no sistema vestibular causam uma síndrome neurológica comum em cães e gatos, denominada síndrome vestibular. Esta pode se apresentar sob a forma periférica e central. A primeira forma envolve receptores periféricos no ouvido interno e a segunda, os núcleos ou tratos do tronco cerebral (TAYLOR, 2003). A otite médiainterna é a causa mais comum de síndrome vestibular periférica em cães e gatos. Além dessa, também há origem idiopática, congênita ou secundária a traumas, infecções e neoplasias (INZANA, 2003). A patofisiologia da síndrome vestibular periférica ainda é pouco conhecida e acredita-se que possa estar relacionada à dinâmica anormal da endolinfa ou neurites da porção vestibular do VIII nervo craniano (SCHUNK; AVERILL, 1983). Agentes infecciosos podem infectar diretamente o ouvido médio e interno, ou a produção de toxinas, provocarem inflamação do labirinto (TAYLOR, 2003). Labirintite linfocítica é outra causa de síndrome vestibular periférica nos cães, porém sua etiologia também não está determinada (FORBES; COOK, 1991). Os sinais de síndrome vestibular periférica desenvolvem-se agudamente e podem ser precedidos por vômitos e náuseas. Os animais podem se apresentar com inclinação de cabeça, nistagmo horizontal ou rotatório não posicional e conjugado, desorientação, paralisia facial, síndrome de Horner, queda e rolamentos (INZANA, 2004). No quadro de origem central ocorre nistagmo posicional e não conjugado, inclinação da cabeça, ataxia simétrica, perda da propriocepção e alterações de estado mental. A vertigem periférica é causada por uma geração desordenada de impulsos nervosos que afetam o aparelho vestibular (LORENZ; KORNEGAY, 2003). O diagnóstico baseia-se nos sinais clínicos, achados de otoscopia, radiografia, tomografia computadorizada ou ressonância magnética do crânio. Muitas vezes os exames complementares não evidenciam nenhuma alteração, levando a um diagnóstico de doença idiopática, todavia pequenas lesões podem não ser evidenciadas (BAYENS-SIMMONDS et al, 1997). Não há tratamento específico para a desordem neurológica, apenas para a causa primária. A recuperação das alterações neurológicas é demorada e, em casos crônicos, os déficits neurológicos podem ser irreversíveis (CHRISMAN, 1985). Os animais afetados podem compensar o déficit neurológico completamente ou permanecerem com leves alterações. Nestes casos, a compensação visual e tátil é muito importante e geralmente precede a recuperação do sistema vestibular, entretanto recidivas podem ocorrer (LEE, 1983). 
A betaistina é uma medicação utilizada em humanos com distúrbio vestibular periférico e até o momento não há relatos de uso clínico em cães. Trata-se de uma molécula, que possui afinidade com os receptores $\mathrm{H}_{3}$ e $\mathrm{H}_{1}$ da histamina, tendo ação antagonista e agonista, respectivamente. Sua ação sobre receptores $\mathrm{H}_{1}$ nos vasos sangüíneos do ouvido interno promove vasodilatação e aumento da permeabilidade, evitando assim a expansão de volume e distensão do sistema hemolinfático. Os receptores $\mathrm{H}_{3}$ encontrados nas membranas pré-sinápticas dos neurônios histaminérgicos e de outros neurônios no cérebro modulam a liberação de histamina e de outros neurotransmissores. Acredita-se que a interação com os receptores $\mathrm{H}_{3}$ pode ser o principal mecanismo através do qual a betaistina exerça seus efeitos clínicos, os quais decorrem do aumento de fluxo sangüíneo no ouvido interno e da redução da atividade emissora dos núcleos vestibulares no cérebro (AFANASYEVA et al, 2003). Com a inibição dos receptores H3, a betaistina induz a um aumento na síntese e liberação de histamina no núcleo tuberomamilar, que por sua vez está conectado ao núcleo vestibular, que desempenha papel fundamental na recuperação de lesões vestibulares (TIGHILET et al, 2005). Acrescenta-se também o efeito da betaistina sobre a dinâmica da endolinfa na compensação do distúrbio vestibular periférico (TIGHILET, et al, 2002; AFANASYEVA et al, 2003). Esta droga também aumenta o fluxo sangüíneo cerebral em outras partes do cérebro, sendo indicada para diversos distúrbios neurológicos de origem central em humanos (MEYER et al, 1974). Em cães anestesiados, a administração de betaistina aumentou o fluxo sangüíneo cerebral, porém o significado clínico não foi explorado (ANDERSON; KUBICEK, 1971; SMITH; MEYER, 1976). Em estudos experimentais, a droga mostrou-se útil na resolução dos sinais vestibulares de felinos domésticos (TIGHILET et al, 1995). Felinos tratados com betaistina apresentaram rápida melhora dos distúrbios vestibulares induzidos (neurectomia vestibular unilateral) em comparação aos animais tratados com placebo (LACOUR, 2000).

Tendo em vista a limitação no tratamento da síndrome vestibular periférica em cães, busca-se com este relatar os efeitos clínicos da utilização do dicloridrato de betaistina em cães com a afecção retro mencionada.

\section{RELATO DE CASO}

Quatro cães com síndrome vestibular periférica atendidos no Hospital Veterinário da UNESP/Jaboticabal foram tratados com dicloridrato de betaistina. Um 
Pastor Alemão, fêmea, de 13 anos, com histórico de incoordenação motora e perda de equilíbrio há um mês e vômitos e sialorréia há dois dias. Ao exame físico, o animal apresentava inclinação da cabeça e andar em círculo para o lado direito, ataxia e nistagmo horizontal. A avaliação otoscópica evidenciou radiopacidade em membrana timpânica direita e ausência de alterações em condutos auditivos. Foram realizados exames hematimétricos (hemograma, atividade sérica de alanina-aminotransferase e fosfatase alcalina, concentração sérica de uréia e creatinina) e radiografia de crânio. A avaliação hematimétrica não revelou alterações, contudo a radiografia de crânio, para avaliação das bulas timpânicas, mostrou aumento de radiopacidade bilateral. Com base no histórico, sinais clínicos e nos resultados dos exames, iniciou-se tratamento para otite média-interna com cefalexina ( $30 \mathrm{mg} / \mathrm{Kg}$ a cada 12 horas, por via oral) e dicloridrato de betaistina $(0,5 \mathrm{mg} / \mathrm{Kg}$ a cada 12 horas, por via oral). Após 21 dias de tratamento, o animal já apresentava melhora clínica sem sinais de perda de equilíbrio, ataxia ou nistagmo, permanecendo apenas com discreta inclinação de cabeça. A avaliação hematológica não evidenciou alterações. Não houve recidiva clínica após a interrupção do tratamento.

O segundo caso descreve uma cadela sem raça definida, de cinco anos, apresentando rolamento para o lado direito, inclinação de cabeça para o mesmo lado, perda de equilíbrio, nistagmo horizontal e incoordenação motora. Realizaram-se hemograma e perfil bioquímico sérico (proteínas totais, albumina, alanina aminotransferase, fosfatase alcalina, uréia e creatinina), que não revelaram alterações. A avaliação otoscópica, citologia do conduto auditivo externo e exame radiográfico simples, para avaliação de bulas timpânicas, também não revelaram alterações dignas de nota. Baseando-se no histórico, sinais clínicos, mesmo com ausência de sinais radiográficos, estabeleceu-se o diagnóstico de síndrome vestibular periférica idiopática. Instituiu-se terapia única com dicloridrato de betaistina $(0,5 \mathrm{mg} / \mathrm{Kg}$ a cada 12 horas), obtendo-se melhora clínica evidente após 10 dias de tratamento e, aos 30 dias, remissão completa dos sinais clínicos. Tanto ao décimo como no trigésimo dia, a avaliação hematológica não evidenciou alterações. Não ocorreu recidiva da síndrome neurológica após a suspensão do tratamento.

O terceiro caso clínico trata de um animal da raça Cocker Spaniel, fêmea, com 11 anos de idade, apresentando inclinação lateral da cabeça de início súbito há aproximadamente 10 dias. Não apresentava ataxia, nistagmo ou outros déficits neurológicos. Sem alterações na otoscopia, citologia do conduto auditivo externo e no 
exame radiográfico de bulas timpânicas. Os exames hematológicos (hemograma, alanina-aminotransferase, fosfatase alcalina, albumina, proteínas totais, uréia e creatinina) encontravam-se dentro dos valores de normalidade para a espécie. Baseado nos achados clínicos estabeleceu-se o diagnóstico de síndrome vestibular periférica idiopática. Administrou-se o dicloridrato de betaistina na dose de $1 \mathrm{mg} / \mathrm{Kg}$ a cada 12 horas. Após uma semana de terapia, o animal apresentou melhora significativa do quadro neurológico. A medicação foi suspensa no décimo dia e o animal não apresentou recidiva. $\mathrm{O}$ perfil hematológico permaneceu inalterado durante o tratamento do animal.

O quarto caso clínico refere-se a um Pastor Belga, macho, com 13 anos, apresentando inclinação da cabeça para o lado esquerdo, andar em círculos e nistagmo com fase rápida ipsilateral e ataxia. Não apresentava alterações em otoscopia, assim como no hemograma e perfil bioquímico (alanina-aminotransferase, fosfatase alcalina, uréia e creatinina). Foi estabelecido diagnóstico de síndrome vestibular geriátrica, sendo instituída terapia com o dicloridrato de betaistina $(0,5 \mathrm{mg} / \mathrm{kg}$ a cada 12 horas $)$. No terceiro dia de tratamento, o cão já não apresentava andar em círculo e nistagmo, apenas permanecia discreta inclinação da cabeça. No décimo dia, o cão não apresentava alteração clínica, sendo a medicação suspensa. Neste momento, a avaliação laboratorial foi repetida, não evidenciando alterações nos parâmetros avaliados. Após a suspensão do tratamento, não houve recidiva do quadro neurológico.

\section{RESULTADOS E DISCUSSÃO}

A síndrome vestibular periférica pode decorrer de fatores ou causas muitas vezes não diagnosticadas por meio de exames laboratoriais. Nos casos relatados, os cães não apresentaram alterações no perfil hematimétrico e citológico do conduto auditivo. Somente o exame radiográfico de um dos cães apresentou alteração, que pode ser decorrente da enfermidade ou mesmo estar relacionada à senilidade do paciente. Os animais que receberam o dicloridrato de betaistina apresentaram melhora significativa do quadro clínico e o tempo de remissão foi curto. Em duas semanas de tratamento, a melhora neurológica já era evidente, independente da causa da síndrome vestibular periférica. A resolução da ataxia e nistagmo deu-se rapidamente, enquanto a inclinação da cabeça se resolveu mais tardiamente. Como os parâmetros hematológicos se 
encontravam dentro dos valores considerados normais, e as avaliações clínicas e laboratoriais não contra-indicavam a utilização da medicação, o cloridrato de betaistina foi prescrito e nenhum efeito adverso ou indesejável, durante o período do tratamento, foi notado. As avaliações hematológicas permaneceram inalteradas ao longo do tratamento, sugerindo ausência de hepatotoxicidade, efrotoxicidade ou hematotoxicidade desta droga em cães. Apesar do tempo de administração da medicação ter sido curto, não houve indícios de comprometimento hepático, renal ou hematológico pelo uso do dicloridrato de betaístina.

Segundo a literatura, a ação sinérgica, entre a estimulação de receptores $\mathrm{H} 1$ e inibição de receptores $\mathrm{H} 3$ são benéficas em pacientes com distúrbios vestibulares e a administração oral de betaistina aumenta o fluxo sangüíneo coclear (MEYER et al, 1974). Este mecanismo está relacionado com a recuperação da função vestibular (TIGHILET et al, 1995). Além disso, a ação antagonista H3 aumenta a síntese e liberação de histamina em porções do sistema vestibular, que desempenham papel fundamental na recuperação de lesões vestibulares (TIGHILET et al, 2002). Desta forma, a betaistina auxilia os mecanismos adaptativos envolvidos na compensação vestibular, tanto dinâmica como estática (TIGHILET et al, 2005). Em felinos, vários estudos comprovam a eficiência da betaistina em acelerar a compensação vestibular (TIGHILET et al, 1995; LACOUR, 2000; TIGHILET et al, 2002; TIGHILET et al, 2005).

As semelhanças entre os distúrbios vestibulares dos animais domésticos e de humanos tornam possível a utilização de algumas terapias comuns. O dicloridrato de betaistina mostrou-se uma excelente opção a ser utilizada isoladamente, ou como adjuvante à terapia para a síndrome vestibular periférica em cães. A persistência de algum grau de inclinação de cabeça é uma conseqüência esperada também em humanos com distúrbios vestibulares tratados com a betaistina (TIGHILET et al, 2002; AFANASYEVA et al, 2003). Segundo Chrisman (1985), em alguns animais, a recuperação pode ser demorada e alguns déficits neurológicos podem permanecer necessitando de intervenção clínica. As causas de desordens vestibulares periféricas são muitas e, em animais idosos, no caso da doença senil, a recuperação pode ser espontânea (INZANA, 2004). Contudo, mesmo nos casos em que ocorre recuperação espontânea, o dicloridrato de betaistina pode ser empregado para acelerar a compensação vestibular, tornando a recuperação mais rápida, como evidenciou LACOUR (2000); TIGHILET et al (2005) em felinos. Recidiva dos sinais clínicos, que 
pode ocorrer em alguns casos (TAYLOR, 2003), não foi registrada nos animais avaliados após um ano de acompanhamento.

\section{CONCLUSÃO}

A rápida remissão do quadro clínico, juntamente com a ausência de alterações clínicas, recidivas e efeitos adversos ao uso da medicação encorajam o uso do dicloridrato de betaistina na terapia de distúrbios vestibulares periféricos em animais de companhia. A satisfação do proprietário frente a uma rápida resposta ao tratamento, também foi um ponto importante observado nestes casos. Apesar de seu emprego na rotina clínica ainda ser incipiente e haver a necessidade de mais estudos para certificar sua eficácia e segurança em animais domésticos, o uso do dicloridrato de betaistina deve ser considerado em casos de síndrome vestibular periférica.

\section{REFERÊNCIAS}

AFANASYEVA, S.A.; GORBACHEVA,F.E.; NATYAZHKINA,G.M. Isolated vertigo: pathogenesis and efficacy of betahistine (Betaserc). Journal of Neurology, Munique, v. 4. p. 12-16, 2003.

ANDERSON, W. D.; KUBICEK, W. G. Effects of Betahistine HCI, Nicotinic Acid, and Histamine on Basilar Blood Flow in Anesthetized Dogs. Stroke, Hagerstown, v.2, p. 409-415, 1971.

BAYENS-SIMMONDS, J.; PURCELL, T.P.; NATION, N.P. Use of magnetic resonance imaging in the diagnosis of central vestibular disease. Can. Vet. J. Ottawa, v. 38, p.38, 1997.

CHRISMAN, C.L. Neurologia dos pequenos animais. São Paulo: Roca, 1985, 432p.

FORBES, S.; COOK, J.R. Congenital peripheral vestibular disease attributed to lymphocytic labyrinthitis in two related litters of Doberman Pinscher pups. J Amer Vet Med Assoc, Schaumburg, v. 198, p. 447-449, 1991.

INZANA, K.D. Distúrbios dos nervos periféricos. In: ETTINGER, S.J.; FELDMANN, E.C. Tratado de medicina interna veterinária . 5.ed. São Paulo: Manole, v.1, cap. $108,2004,2256 p$. 
INZANA, K. D. Síndromes Vestibulares. In: PELLEGRINO, F.C.; SURANITI, A.; GARIBALDI, L. EI libro de Neurologia para la practica clinica. Buenos Aires: Intermédica, cap. 15, 2003, 720p.

LACOUR, M. Vestibular compensation in the cat: The Role of the Histaminergic System. Acta Oto-Laryngologica. Estocolmo, v. 120, p.15-18, 2000.

LEE, M. Congenital vestibular disease in a German shepherd dog. Vet Rec. Londres, v.113, p. 571, 1983.

LORENZ, M.D.; KORNEGAY, J.N. Oliver \& Lorenz's Handbook of Veterinary Neurology, Philadelphia: W.B.Saunders, 2003, 480p.

MEYER, J. S. et al. Orally Administered Betahistine and Regional Cerebral Blood Flow in Cerebrovascular Disease. J. Clin. Pharmacol. Thousand Oaks, v. 14, p. 280289, 1974.

SCHUNK, K.L.; AVERILL, D.R. Peripheral vestibular syndrome in the dog: A review of 83 cases. J Amer Vet Med Assoc. Schaumburg, v.182, p. 1354-1357, 1983.

SMITH, K. A.; MEYER, M. W. Distribution of cardiac output in dogs during intravenous infusion of betahistine. Stroke. Hagerstown, v.7, p. 257-260, 1976.

TAYLOR, S.M. Head Tilt. In: NELSON, R.W., COUTO, C.G. Small Animal Internal Medicine, 3. ed. St. Louis: Mosby,. Cap. 70, 2003, 953p.

TIGHILET, B.; LEONARD, J.; LACOUR, M. Betahistine Dihydrochloride treatment facilitates vestibular compensation in the cat. J Vet Res, Schaumburg, v. 5, p. 53-66, 1995.

TIGHILET, B.; TROTTIER, B.; LACOUR, M. Dose- and duration-dependent effects of betahistine dihydrochloride treatment on histamine turnover in the cat. Eur. J. Pharmacol; Utrecht, v. 523, p. 54-63, 2005.

TIGHILET, B et al. Betahistine dihydrochloride interaction with the histaminergic system in the cat: neurochemical and molecular mechanisms. Eur. J. Pharmacol. Utrecht, v. 20, p. 63-73, 2002. 